\title{
The Feasibility of Using Virtual Reality Technology in Better Dental Education at Kermanshah Dental School
}

\author{
Meysam Siyah Mansoory (iD) ${ }^{1}$, Danial Yousefi ${ }^{2}$, Vida Sepahi (iD) ${ }^{3}$ and Hedaiat Moradpoor (iD) ${ }^{4}{ }^{*}$ \\ ${ }^{1}$ Department of Biomedical Engineering, School of Medicine, Kermanshah University of Medical Sciences, Kermanshah, Iran \\ ${ }^{2}$ Department of Computer Engineering, Islamic Azad University of Central Tehran Branch, Tehran, Iran \\ ${ }^{3}$ Education Development Center, Kermanshah University of Medical Sciences, Kermanshah, Iran \\ ${ }^{4}$ Department of Prosthodontics, School of Dentistry, Kermanshah University of Medical Sciences, Kermanshah, Iran \\ "Corresponding author: Department of Prosthodontics, School of Dentistry, Kermanshah University of Medical Sciences, Kermanshah, Iran. Email: \\ hedaiat.moradpoor@gmail.com
}

Received 2018 November 03; Revised 2018 December 24; Accepted 2018 December 30.

Keywords: Virtual Reality, Education, Dental School

\section{Dear Editor,}

Dentistry is one of the academic disciplines in which experience and practical work is essential for schooling. Nowadays, dental schools in all over the world face major challenges in providing educational services to meet the students' eligibility (1). Due to the position of this field and its impact on oral health and hygiene, providing a virtual environment for teaching and practicing practical dentistry courses in the best universities of the world has been considered.

Virtual reality (VR) is a new technology that allows the user to interact with a computerized simulation environment. Because of complexity in software and hardware developing of VR, this technology in education has not been used by professors and students in Iran's dental school. Given the different nature of dentistry and the urgent need to visualize education, VR technology is one of the best choices for creating an environment for practical experience in dentistry (2).

Approximately 1,000 to 1,100 people annually accept to dental universities in Iran. Considering the dangers and consequences of using the patient and the cost of dental materials and equipment, it is necessary to implement a suitable VR environment which is the most similar to the practical environment of activities and provide students the most realistic experience. The use of VR in addition to creating gravity for students and providing better education for them, greatly reduces the risks of using the patient and the financial costs of dental materials and equipment provided by the medical universities (3).

This educational product is based on VR technology, in which the students are introduced into the virtual world by putting VR headset on their head while prosthodontics specialist teaches the procedure on the dental phantom. Unlike traditional education in which the student attends the classroom with other students and may not have a proper view while the teacher works on an educational phantom, students can control their viewing angle in VR by turning head or touching the controller buttons. This will allow the students to see how the specialist works on the phantom at all angles and do not miss any educational point. After training with this system, learningrelated questionnaires are acquired and statistical analysis is done.

New studies indicate that VR technology is a valuable tool in the pre-clinical dental education that can augment the traditional teaching methods. Furthermore, the proximity of the VR environment to student's interests will trigger positive impacts on dental students. Rapid developments in hardware and software technology can lead to a better VR experience and adaptation of this technology as a necessary part of modern education. For the effectiveness of VR in dentistry, the design of the environment should be based on specialist's comments and provide the possibility of creating immediate feedback and interactive and motivational environments.

The researchers designed a VR technology for teaching neutral zone application skill and fixing artificial teeth in complete denture prosthodontics. In order to evaluate students' satisfaction with the VR-assisted course, a researcher-built questionnaire which was based on Likert scale (5 options) was used. The questionnaire was reviewed and modified multiple times and the validity of the ques- 
tionnaire was confirmed by 10 experts from the faculty members of dentistry department. The reliability of the questionnaire was calculated by Cronbach's alpha coefficient as $76 \%$. Twenty-four students (96\%) believed that using this technology helped them learning the content of the course and 19 (76\%) of them were fully satisfied with the use of this technology.

\section{Footnotes}

Conflicts of Interests: The authors have not disclosed any potential conflicts of interest.
Funding/Support: None declared.

\section{References}

1. Roy E, Bakr MM, George R. The need for virtual reality simulators in dental education: A review. Saudi Dent J. 2017;29(2):41-7. doi: 10.1016/j.sdentj.2017.02.001. [PubMed: 28490842]. [PubMed Central: PMC5411891].

2. Huang TK, Yang $\mathrm{CH}$, Hsieh YH, Wang JC, Hung CC. Augmented reality (AR) and virtual reality (VR) applied in dentistry. Kaohsiung J Med Sci. 2018;34(4):243-8. doi: 10.1016/j.kjms.2018.01.009. [PubMed: 29655414]

3. Kalpana D, Rao SJ, Venkatesh P, Bhat P. Virtual articulartors: Reality in virtuality-a review. Int J Sci Res. 2018;7(1). 